\title{
Immediate impact of exercise on arterial stiffness in humans
}

\author{
Jae-Bin Seo, Woo-Young Chung, Sang-Hyun Kim, Myung-A Kim, Joo-Hee Zo ${ }^{*}$ \\ Division of Cardiology, Department of Internal Medicine, Seoul National University Boramae Medical Center/Seoul National Uni- \\ versity College of Medicine, Seoul, Korea \\ Email: *jooheezo@hanmail.net
}

Received 15 October 2012; revised 22 November 2012; accepted 30 November 2012

\begin{abstract}
Background and objectives: Increased arterial stiffness is an accepted cardiovascular risk factor. Several studies have shown that regular exercise is associated with reduced arterial stiffness. However, the acute effect is not well known. The aim of this study was to evaluate the immediate impact of exercise on arterial stiffness measured by pulse wave velocity (PWV). Subjects and Methods: Data from 100 consecutive patients who underwent graded maximal aerobic exercise test and pulse wave velocity study due to chest pain were analyzed. Results: With respect to brachial-ankle pulse wave velocity (baPWV), it decreased after the exercise test (before vs. after; $1420 \pm 260$ vs $1358 \pm 245,1428 \pm 255$, vs $1360 \pm 239 \mathrm{~cm} / \mathrm{sec}$; left and right, respectively). Upper extremity systolic blood pressure did not change from baseline. However, upper extremity diastolic blood pressure increased slightly. In addition, lower extremity blood pressure decreased in both systolic and diastolic phases. In both normotensives and hypertensives, baPWV decreased after exercise test. Conclusion: Exercise immediately decreases arterial stiffness.
\end{abstract}

Keywords: Arterial Stiffness; Pulse Wave Velocity

\section{INTRODUCTION}

Cardiovascular disease is an increasing cause of death. Increased arterial stiffness is an accepted risk factor for atherosclerosis and cardiovascular disease [1,2]. This also has been known to be associated with aging process, leading to isolated systolic hypertension $[3,4]$. Therefore, decreasing arterial stiffness might be one of the important therapeutic targets to prevent cardiovascular disease.

Exercise is associated with improved arterial stiffness as demonstrated by several studies [5-10]. Actually, there are many forms of exercise. Among them, regular aero-

*Corresponding author. bic exercise gives a rise to lower level of arterial stiffness in humans, compared with that of sedentary persons [11]. Moreover, a single bout of cycling exercise acutely decreases arterial stiffness [12]. However, the immediate impact of exercise on arterial stiffness is not well studied.

The purpose of this study was to elucidate the immediate impact of running exercise on arterial stiffness. We founded the hypothesis that running exercise would decrease arterial stiffness immediately. To test our hypothesis, we analyzed brachial-ankle pulse wave velocity (baPWV), an index of arterial stiffness, which is measured before and after graded maximal aerobic exercise test (Treadmill test).

\section{METHODS}

\subsection{Subjects}

This study included one hundred consecutive patients who underwent graded maximal aerobic exercise test and pulse wave velocity study due to chest pain at Boramae Medical Center between September 2008 and February 2009. The patients underwent PWV measurement before and immediately after treadmill test. The time gap was within 10 minutes between stopping of exercise and the measurement of PWV to investigate the immediate effect of exercise on PWV.

\subsection{Measurement of Brachial-Ankle Pulse Wave Velocity}

The PWV was measured by a VP-2000 (Colin Co. Ltd, Komaki, Japan). Using this machine, we could measure PWV, blood pressure of both extremities, electrocardiogram and phonocardiogram (PCG) at once. The electrocardiogram electrodes were attached to both wrists, which made us to get lead II electrocardiogram. PCG sensor was usually located at the left sternal border of the level of 4th rib. Cuffs were encircled around both arms and ankles, which were connected to plethysmographic sensor and oscillometric sensor. The plethysmographic sensor determined pulse volume recording and the oscillometric 
sensor determine the blood pressure. Pulse wave velocity (PWV) is defined as the distance between two distinct points $(\mathrm{cm})$ divided by the pulse wave transit time $(\mathrm{sec})$. Accordingly, baPWV was defined as the distance between brachial artery and each side of ankles divided by the pulse wave transit time. In our study, brachial-ankle PWV (baPWV) was evaluated. Right and Left baPWV was calculated from the distance between right brachial artery and each side of ankles respectively. The distance was automatically calculated by patient's height based on statistical studies. The brachial-ankle pulse transit time was measured from the ascending points of the right brachial pulse volume recording and each ankle pulse volume recordings. All the measurements were acquired during regular sinus rhythm.

\subsection{Running Exercise}

All patients, complaining of chest pain, were tested using the standard symptom-limited Bruce's treadmill protocol with a commercially available system. All patients were instructed not to eat, drink caffeinated beverages, or smoke any kind of tobacco for $3 \mathrm{~h}$ before testing. After the baseline 12-lead ECG was recorded, the patient started exercise on treadmill. The 12-lead ECG, heart rate response and blood pressure were recorded every $3 \mathrm{~min}$ utes. If a patient showed abnormal symptoms or signs (moderate to severe angina, a drop in systolic blood pressure of $>10 \mathrm{mmHg}$ from baseline, cyanosis or pallor, syncope, severe dyspnea, sustained ventricular tachycardia, severe ischemic ST depression or ST elevation $\geq 1.0$ $\mathrm{mm}$ in noninfarct leads without diagnostic Q waves) or the target heart rate was achieved, the treadmill test and recording were stopped. The results of the treadmill exercise were interpreted by cardiologists.

For the interpretation, the PQ junction was chosen as the isoelectric point. The development of $0.10 \mathrm{mV}$ (1 $\mathrm{mm}$ ) or greater of the $\mathrm{J}$ point depression measured from the PQ junction, with a horizontal type or down-sloped type of ST segment, depressed $\geq 0.10 \mathrm{mV}$ for $80 \mathrm{~ms}$ after the J point (ST 80) in three consecutive beats with a stable baseline, was considered an abnormal response. When the ST 80 measurement was difficult to determine at rapid heart rates, the ST 60 measurement was used. If the patient did not reach $85 \%$ of predicted maximal heart rate (target heart rate) adjusted for age, we defined the result as indeterminate.

The New York Heart Association (NYHA) functional class was also recorded for each patient according to the achieved metabolic equivalents (METs). One MET is equivalent to $3.5 \mathrm{~mL}$ of $\mathrm{O}^{2} / \mathrm{kg}$ of body weight per min. NYHA functional class I was defined as METs $\geq 7$; class II as METs between 5 and 6; and class III as METs between 2 and 4. NYHA functional classes II and III were considered impaired exercise capacity.

\subsection{Statistical Analysis}

Statistical analysis was performed with the use of the SPSS/PC software package (SPSS version 12.0). Statistical differences among the groups for the continuous variable were evaluated using one-way ANOVA. Data are expressed as means \pm SDs. $p<0.05$ was considered statistically significant.

\section{RESULTS}

The baseline characteristics of study group are presented in Table 1. The patients $(n=100)$ were $53.4 \pm 13.1$ years old, and $60 \%$ were men. The height was $163.3 \pm 8.6 \mathrm{~cm}$ and the weight was $65.9 \pm 10.3 \mathrm{~kg}$. Furthermore, body mass index (BMI) was $24.6 \pm 3.1 \mathrm{~kg} / \mathrm{m}^{2}$. Therefore, relatively obese patients were included in this study.

The exercise time was $9.1 \pm 2.1$ minutes and exercise METs was $10.7 \pm 2.2$. Therefore, the patients were considered to undergo graded exercise test adequately.

The systolic and diastolic arterial blood pressures were significantly increased after graded maximal aerobic exercise test (Table 2). Moreover, the heart rate was also increased significantly after the test. However, the pulse pressures were significantly decreased after the test. Brachial-ankle pulse wave velocity before and after the exercise is demonstrated in Table 3. The right baPWV was $1419.8 \pm 260.4 \mathrm{~cm} / \mathrm{sec}$ before exercise and $1358.1 \pm$ $244.8 \mathrm{~cm} / \mathrm{sec}$ after exercise. Similarly, the left baPWV was $1427.8 \pm 255.4 \mathrm{~cm} / \mathrm{sec}$ before exercise and $1360.1 \pm$ $238.9 \mathrm{~cm} / \mathrm{sec}$ after exercise. The differences were statistically significant for both right and left baPWV values. The PWV of hypertensives was higher than normotensives both before and after exercise. Moreover, in both groups, the PWV decreased after a bout of running exercise (Table 4). In addition, both high-intensity $(\geq 10.9$ METs; 50 of 100 patients) and low-intensity (<10.9 METs) exercise decreased right and left baPWV values (Table 5). The PWV was higher in the patients of low-intensity exercise.

\section{DISCUSSION}

In the present study, we investigated the immediate impact

Table 1. Anthropometric data of study group.

\begin{tabular}{cc}
\hline Anthropometric data & Values \\
\hline Number & 100 \\
Male:Female & $60: 40$ \\
Age (year) & $53.4 \pm 13.1$ \\
Height $(\mathrm{cm})$ & $163.3 \pm 8.6$ \\
Weight $(\mathrm{Kg})$ & $65.9 \pm 10.3$ \\
BMI $\left(\mathrm{Kg} / \mathrm{m}^{2}\right)$ & $24.6 \pm 3.1$ \\
\hline
\end{tabular}


Table 2. Arterial blood pressure, pulse pressure and heart rate before and after exercise ( $\mathrm{mmHg}$ ).

\begin{tabular}{cccc}
\hline & Baseline & Post-exercise & p-value \\
\hline Right brachial systolic BP & $127.9 \pm 13.3$ & $126.6 \pm 12.2$ & 0.093 \\
Right brachial diastolic BP & $77.5 \pm 10.0$ & $79.2 \pm 9.5$ & 0.002 \\
Right brachial pulse pressure & $50.4 \pm 8.7$ & $47.4 \pm 8.7$ & $<0.001$ \\
Left brachial systolic BP & $126.6 \pm 13.3$ & $126.0 \pm 12.3$ & 0.300 \\
Left brachial diastolic BP & $77.0 \pm 9.8$ & $78.3 \pm 8.8$ & 0.006 \\
Left brachial pulse pressure & $49.6 \pm 9.4$ & $47.7 \pm 8.6$ & 0.001 \\
Right ankle systolic BP & $143.8 \pm 17.6$ & $135.0 \pm 17.5$ & $<0.001$ \\
Right ankle diastolic BP & $74.9 \pm 9.5$ & $71.5 \pm 9.6$ & $<0.001$ \\
Right ankle pulse pressure & $68.9 \pm 14.1$ & $63.5 \pm 12.6$ & $<0.001$ \\
Left ankle systolic BP & $144.5 \pm 17.3$ & $135.3 \pm 17.3$ & $<0.001$ \\
Left ankle diastolic BP & $76.4 \pm 13.9$ & $71.9 \pm 9.1$ & 0.001 \\
Left ankle pulse pressure & $68.1 \pm 17.0$ & $63.4 \pm 13.1$ & 0.001 \\
Heart rate (bpm) & $68.3 \pm 9.0$ & $81.0 \pm 9.8$ & $<0.001$ \\
\hline
\end{tabular}

Table 3. Pulse wave velocity before and after exercise.

\begin{tabular}{cccc}
\hline & Baseline & Post-exercise & p-value \\
\hline Right baPWV $(\mathrm{cm} / \mathrm{sec})$ & $1419.8 \pm 260.4$ & $1358.1 \pm 244.8$ & $<0.001$ \\
Left baPWV $(\mathrm{cm} / \mathrm{sec})$ & $1427.8 \pm 255.4$ & $1360.1 \pm 238.9$ & $<0.001$ \\
\hline
\end{tabular}

Table 4. Pulse wave velocity before and after exercise in normotensives and hypertensives.

\begin{tabular}{lcccc}
\hline & & Baseline & Post-exercise & p-value \\
\hline \multirow{2}{*}{ Normotensives } & Right baPWV $(\mathrm{cm} / \mathrm{sec})$ & $1315.1 \pm 215.7$ & $1278.9 \pm 233.5$ & 0.009 \\
& Left baPWV $(\mathrm{cm} / \mathrm{sec})$ & $1330.0 \pm 217.7$ & $1289.6 \pm 231.4$ & 0.002 \\
\multirow{2}{*}{ Hypertensives } & Right baPWV $(\mathrm{cm} / \mathrm{sec})$ & $1516.5 \pm 262.4$ & $1431.2 \pm 233.9$ & $<0.001$ \\
& Left baPWV $(\mathrm{cm} / \mathrm{sec})$ & $1518.1 \pm 256.2$ & $1426.6 \pm 228.6$ & $<0.001$ \\
\hline
\end{tabular}

Table 5. Pulse wave velocity before and after exercise in high-intensity and low-intensity exercise.

\begin{tabular}{lcccc}
\hline & & Baseline & Post-exercise & p-value \\
\hline \multirow{2}{*}{ High-intensity } & Right baPWV $(\mathrm{cm} / \mathrm{sec})$ & $1328.4 \pm 192.9$ & $1277.8 \pm 210.9$ & $<0.001$ \\
& Left baPWV $(\mathrm{cm} / \mathrm{sec})$ & $1340.4 \pm 197.2$ & $1279.7 \pm 213.6$ & $<0.001$ \\
\multirow{2}{*}{ Low-intensity } & Right baPWV $(\mathrm{cm} / \mathrm{sec})$ & $1511.2 \pm 287.6$ & $1438.4 \pm 251.8$ & 0.001 \\
& Left baPWV $(\mathrm{cm} / \mathrm{sec})$ & $1515.1 \pm 278.1$ & $1442.0 \pm 237.1$ & $<0.001$ \\
\hline
\end{tabular}

of exercise on pulse wave velocity which is the representative of arterial stiffness. We found that PWV decreased immediately after a single exercise session in humans. The difference of blood pressure reduction between upper extremities and lower extremities could be explained by the different exercise load on upper body and lower body during treadmill test.

Arterial stiffness is in inverse relationship with arterial compliance. Increased arterial stiffness is a risk factor for the atherosclerosis and cardiovascular disease $[1,2]$. There- fore, it is important to take measures to reduce arterial stiffness including exercise. Moreover, the pathophysiologic phenomenon of increased arterial stiffness underlies several pathologic processes including hypertension, left ventricular hypertrophy, myocardial infarction, and congestive heart failure [13]. Life style modifications are recommended in several important diseases, which include hypertension, diabetes and dyslipidemia. Exercise is an important component of life style modifications. There are two diverse types of exercise. One is 
endurance exercise and the other is strength exercise. Previous investigators have demonstrated that endurance exercise decreases arterial stiffness [6,14-16], whereas strength exercise increases arterial stiffness [16-20].

PWV is known to be traditional index of arterial stiffness and established index of arterial stiffness in adults $[2,21]$. Stiffness of the central elastic arteries, which is translated into traditional carotid-femoral PWV, but not of the peripheral muscular arteries, is an independent cardiovascular risk factor. We measured baPWV and this involves both central and peripheral arterial stiffness. Nonetheless, according the study of Sugawara et al. [22], the aortic and leg pulse wave velocity were correlated with baPWV independently, accounting for 58\% and $23 \%$ of the total variance in baPWV respectively.

Our results agree with previous study by Kingwell et $a l$. , in that arterial stiffness decreases after aerobic exercise [12]. This study investigated the effects of a single bout of 30 -min cycling at $65 \%$ of maximal oxygen uptake on whole body arterial compliance. Whole body arterial compliance was elevated at 30 -min postexercise $(66 \% \pm 26 \%)$, followed by a decline to baseline 1 -hour after exercise. Aortic PWV, which is inversely related to compliance, was reduced $(4 \% \pm 2 \%)$. PWV in the leg decreased by $10 \% \pm 4 \%$ at this time. The noteworthy finding of the present study is as follows: acute single session of aerobic graded exercise decreased arterial stiffness which is measured by baPWV. Combined with the findings of Kingwell et al., the favorable effect of aerobic exercise on arterial stiffness is immediate and persists up to 1-hour. In terms of laterality, both left and right baPWV decreased. This result is consistent with the studies of Heffernan et al. and Sugawara et al. [23,24]. They demonstrated that that regional exercise (single-leg press or single-leg cycling) affects only the regional artery. Our subjects did the exercise which involved bilateral legs.

What is the mechanism of reduced arterial stiffness after aerobic exercise? The purpose of the present study was only to determine whether aerobic exercise affects arterial stiffness immediately (i.e. within 10 minutes), therefore, we could not find the answer with this study itself. Basically, the mechanism might not be different from that of acute decrease of PWV, which has been shown in previous studies. Hence, we could infer the mechanism by those studies.

Kingwell et al. suggested that acute increase of arterial compliance after exercise might be related to vasodilation of the exercising muscle vascular beds [12]. However, they found that the mechanisms responsible for the alterations in arterial compliance are not related to changes in blood pressure nor is the time course of the response. Instead, relaxation of vascular smooth muscle, which transfers stress from the less extensive collagen fibers to elastin, might be likely to account for the acute elevation in compliance [12,25]. For the vasodilation, they raised the possibility that vasodilatory metabolites might account for peripheral dilation during and immediately after exercise in the exercising limb. Sugawara et al. performed the study to test the hypothesis that the exercise-induced decrease in arterial stiffness is caused by the increased production of NO in vascular endothelium with exercise [24]. This study used the method of NO synthase blockade. However, the NO synthase blockade $\times$ time interaction on exercised leg PWV was not significant. Therefore, they concluded that increased production of NO might not be a major factor in the decrease of arterial stiffness with short-duration aerobic exercise. Furthermore, sympathoinhibition has been documented after aerobic exercise and, therefore, implicated as a feasible mechanism underlying the reduction of arterial stiffness [12].

Is immediate reduction of arterial stiffness a beneficial effect on long-term arterial stiffness? Cameron et al. investigated the effect of 4 weeks of exercise training on total systemic arterial compliance [5]. They found that 1) exercise training increases systemic arterial compliance; 2) that the increase of systemic arterial compliance is greater than that due to changes in blood pressure and is likely to include a component due to change in intrinsic arterial compliance; and 3) that the induced change in $\mathrm{SAC}$ is linearly related to change in $\mathrm{VO}_{2}$ max. Furthermore, Tanaka et al. demonstrated that regular aerobicendurance exercise attenuates age-related reductions in central arterial compliance and restores levels in previously sedentary healthy middle-aged and older men [6]. Besides these studies, there have been several studies showing that baseline arterial stiffness is decreased by aerobic exercise training [7-10]. Taken together, although the effect of a single bout of aerobic exercise is slight, long-term exercise training shows favorable outcomes on arterial stiffness and repetition of immediate reduction in arterial stiffness decreases baseline levels of arterial stiffness.

The PWV velocity was higher in hypertensives, which is consistent with the study that hypertension was associated with increased arterial stiffness [26]. In addition, our results suggested that a bout of exercise is beneficial to both normotensives and hypertensives.

There were several limitations in this study. First, the information about concomitant disease status could not be gathered completely, which might be confounding factors, because many subjects did not undergo other tests besides treadmill test and PWV. Second, we analyzed the data from relatively small number of patients in a single hospital. Third, we studied a group of patients who were referred for the work-up for suspected ischemic heart disease. In other words, the cohort might involve 
the subjects of both healthy vascular function and vascular dysfunction. And the final limitation was the lack of comparison of the effect of treadmill test and other forms of exercise. In other words, we only showed the effect of aerobic exercise on arterial stiffness. However, one would whether anaerobic exercise plays differently in arterial stiffness. Therefore, it is requested to perform the study to compare the effect of aerobic and anaerobic exercise.

\section{CONCLUSION}

Arterial stiffness immediately decreases after aerobic exercise. Furthermore, we propose the possibility that this effect could be due to vascular dilatation of vasodilator and sympathoinhibition and this immediate effect of exercise could reduce baseline arterial stiffness in the end.

\section{REFERENCES}

[1] Laurent, S., Boutouyrie, P., Asmar, R., Gautier, I., Laloux, B., Guize, L., Ducimetiere, P. and Benetos, A. (2001) Aortic stiffness is an independent predictor of all-cause and cardiovascular mortality in hypertensive patients. Hypertension, 37, 1236-1241. doi:10.1161/01.HYP.37.5.1236

[2] Blacher, J., Asmar, R., Djane, S., London, G.M. and Safar, M.E. (1999) Aortic pulse wave velocity as a marker of cardiovascular risk in hypertensive patients. Hypertension, 33, 1111-1117. doi:10.1161/01.HYP.33.5.1111

[3] Franklin, S.S., Khan, S.A., Wong, N.D., Larson, M.G. and Levy, D. (1999) Is pulse pressure useful in predicting risk for coronary heart Disease? The Framingham heart study. Circulation, 100, 354-360. doi:10.1161/01.CIR.100.4.354

[4] Schiffrin, E.L. (2004) Vascular stiffening and arterial compliance: Implications for systolic blood pressure. American Journal of Hypertension, 17, 39S-48S. doi:10.1016/j.amjhyper.2004.08.019

[5] Cameron, J.D. and Dart, A.M. (1994) Exercise training increases total systemic arterial compliance in humans. American Journal of Physiology, 266, H693-H701.

[6] Tanaka, H., Dinenno, F.A., Monahan, K.D., Clevenger, C.M., DeSouza, C.A. and Seals, D.R. (2000) Aging, habitual exercise, and dynamic arterial compliance. Circulation, 102, 1270-1275. doi:10.1161/01.CIR.102.11.1270

[7] Otsuki, T., Maeda, S., Iemitsu, M., Saito, Y., Tanimura, Y., Ajisaka, R., et al. (2007) Relationship between arterial stiffness ad athletic training programs in young adult men. American Journal of Hypertension, 20, 967-973. doi:10.1016/j.amjhyper.2007.05.001

[8] Otsuki, T., Maeda, S., Iemitsu, M., Saito, Y., Tanimura, Y., Ajisaka, R., et al. (2007) Vascular endothelium-derived factors and arterial stiffness in strength- and endurance-trained men. American Journal of Physiology-Heart and Circulatory Physiology, 292, H786-H791. doi:10.1152/ajpheart.00678.2006

[9] Kingwell, B.A., Cameron, J.D., Gillies, K.J., Jennings, G.L. and Dart, A.M. (1995) Arterial compliance may in- fluence baroreflex function in athletes and hypertensives. American Journal of Physiology, 268, H411-H418.

[10] Schmidt-Trucksäss, A., Schmid, A., Brunner, C., Scherer, N., Zäch, G., Keul, J., et al. (2000) Arterial properties of the carotid and femoral artery in endurance-trained and paraplegic subjects. Journal of Applied Physiology, 89, 1956-1963.

[11] Okamoto, T., Masuhara, M. and Ikuta, K. (2007) Combined aerobic and resistance training and vascular function: Effect of aerobic exercise before and after resistance training. Journal of Applied Physiology, 103, 1655-1661. doi:10.1152/japplphysiol.00327.2007

[12] Kingwell, B.A., Berry, K.L., Cameron, J.D., Jennings, G.L. and Dart, A.M. (1997) Arterial compliance increases after moderate-intensity cycling. American Journal of Physiology, 273, H2186-H2191.

[13] Safar, M.E. and London, G.M. (2000) Therapeutic studies and arterial stiffness in hypertension: Recommendations of the European Society of Hypertension. The Clinical Committee of Arterial Structure and Function. Working Group on Vascular Structure and Function of the European Society of Hypertension. Journal of Hypertension, 18, 1527-1535. doi:10.1097/00004872-200018110-00001

[14] Kakiyama, T., Sugawara, J., Murakami, H., Maeda, S., Kuno, S. and Matsuda, M. (2005) Effects of short-term endurance training on aortic distensibility in young males. Medicine \& Science in Sports \& Exercise, 37, 267-271. doi:10.1249/01.MSS.0000152733.12578.5A

[15] Sugawara, J., Otsuki, T., Tanabe, T., Hayashi, K., Maeda, S. and Matsuda, M. (2006) Physical activity duration, intensity, and arterial stiffening in postmenopausal women. American Journal of Hypertension, 19, 1032-1036. doi:10.1016/j.amjhyper.2006.03.008

[16] Otsuki, T., Maeda, S., Iemitsu, M., Saito, Y., Tanimura, Y., Ajisaka, R., et al. (2006) Effects of athletic strength and endurance exercise training in young humans on plasma endothelin-1 concentration and arterial distensibility. Experimental Biology and Medicine, 231, 789-793.

[17] Miyachi, M., Kawano, H., Sugawara, J., Takahashi, K., Hayashi, K., Yamazaki, K., et al. (2004) Unfavorable effects of resistance training on central arterial compliance: A randomized intervention study. Circulation, 110, 28582863. doi:10.1161/01.CIR.0000146380.08401.99

[18] Miyachi, M., Donato, A.J., Yamamoto, K., Takahashi, K., Gates, P.E., Moreau, K.L., et al. (2003) Greater age-related reductions in central arterial compliance in resistance-trained men. Hypertension, 41, 130-135. doi:10.1161/01.HYP.0000047649.62181.88

[19] Bertovic, D.A., Waddell, T.K., Gatzka, C.D., Cameron, J.D., Dart, A.M. and Kingwell, B.A. (1999) Muscular strength training is associated with low arterial compliance and high pulse pressure. Hypertension, 33, 13851391. doi:10.1161/01.HYP.33.6.1385

[20] Cortez-Cooper, M.Y., DeVan, A.E., Anton, M.M., Farrar, R.P., Beckwith, K.A., Todd, J.S., et al. (2005) Effects of high intensity resistance training on arterial stiffness and wave reflection in women. American Journal of Hypertension, 18, 930-934. doi:10.1016/j.amjhyper.2005.01.008 
[21] Chuang, S.Y., Chen, C.H., Cheung, C.M. and Chou, P. (2005) Combined use of brachial-ankle pulse wave velocity and ankle-brachial index for fast assessment of arteriosclerosis and atherosclerosis in a community. International Journal of Cardiology, 98, 99-105.

doi:10.1016/j.ijcard.2004.01.019

[22] Sugawara, J., Hayashi, K., Yokoi, T., Cortez-Cooper, M.Y., DeVan, A.E., Anton, M.A., et al. (2005) Brachialankle pulse wave velocity: An index of central arterial stiffness? Journal of Human Hypertension, 19, 401-406. doi:10.1038/sj.jhh.1001838

[23] Heffernan, K.S., Rossow, L., Jae, S.Y., Shokunbi, H.G., Gibson, E.M. and Fernhall, B. (2006) Effect of single-leg resistance exercise on regional arterial stiffness. European Journal of Applied Physiology, 98, 185-190. doi:10.1007/s00421-006-0259-9
[24] Sugawara, J., Maeda, S., Otsuki, T., Tanabe, T., Ajisaka, R. and Matsuda, M. (2004) Effects of nitric oxide synthase inhibitor on decrease in peripheral arterial stiffness with acute low-intensity aerobic exercise. American Journal of Physiology-Heart and Circulatory Physiology, 287, H2666-H2669. doi:10.1152/ajpheart.00077.2004

[25] Belz, G.G. (2001) Elastic properties and windkessel function of the human aorta. Cardiovascular Drug and Therapy, 9, 73-83. doi:10.1007/BF00877747

[26] Avolio, A.P., Deng, F.Q., Li, W.Q., Luo, Y.F., Huang, Z.D., Xing, L.F., et al. (1985) Effects of aging on arterial distensibility in populations with high and low prevalence of hypertension: Comparison between urban and rural communities in China. Circulation, 71, 202-210.

doi:10.1161/01.CIR.71.2.202 